\title{
MRI evaluation prior to Transcatheter Aortic Valve Implantation (TAVI): When to acquire and how to interpret
}

\author{
Abhishek Chaturvedi ${ }^{1}$ - Susan K. Hobbs ${ }^{1}$ • Fred S. Ling ${ }^{2}$ - Apeksha Chaturvedi ${ }^{1}$. \\ Peter Knight ${ }^{3}$
}

Received: 6 July 2015 /Revised: 7 January 2016 / Accepted: 26 January 2016 / Published online: 25 February 2016

(C) The Author(s) 2016. This article is published with open access at Springerlink.com

\begin{abstract}
Transcatheter Aortic Valve Implantation (TAVI) is increasingly being used in patients with severe aortic stenosis who are not candidates for surgery. ECG-gated $\mathrm{CT}$ angiography (CTA) plays an important role in the preoperative planning for these devices. As the number of patients undergoing these procedures increases, a subset of patients is being recognized who have contraindications to iodinated contrast medium, either due to a prior severe allergic type reaction or poor renal function. Another subgroup of patients with low flow and low gradient aortic stenosis is being recognized that are usually assessed for severity of aortic stenosis by stress echocardiography. There are contraindications to stress echocardiography and some of these patients may not be able to undergo this test. Non-contrast MRI can be a useful emerging modality for evaluating these patients. In this article, we discuss the emerging indications of noncontrast MRI in preoperative assessment for TAVI and describe the commonly used MRI sequences. A comparison of the most important measurements obtained for
\end{abstract}

Electronic supplementary material The online version of this article (doi:10.1007/s13244-016-0470-0) contains supplementary material, which is available to authorized users.

Abhishek Chaturvedi

toabhic@gmail.com

1 Department of Imaging Sciences, University of Rochester Medical Center, 601 Elmwood Ave, P.O. Box no. 648, Rochester, NY 14642, USA

2 Department of Medicine, Cardiology, University of Rochester Medical Center, Rochester, NY, USA

3 Department of Surgery, Cardiac, University of Rochester Medical Center, Rochester, NY, USA
TAVI assessment on CTA and MRI from same subjects is included.

\section{Teaching Points}

- MRI can be used for preoperative assessment of aortic annulus.

- MRI is an alternate to CTA when iodinated contrast is contraindicated.

- Measurements obtained by non-contrast MRI are similar to contrast enhanced CTA.

- MRI can be used to assess severity of aortic stenosis.

Keywords Transcatheter aortic valve implantation . Transcatheter aortic valve replacement $\cdot$ Magnetic resonance imaging $\cdot$ Severe aortic stenosis $\cdot$ Non-contrast MRI

\section{Background}

Valvular heart disease is estimated to account for as many as $20 \%$ of cardiac surgical procedures performed in the United States [1]. Transcatheter aortic valve implantation (TAVI) has become an increasingly promising treatment for high surgical risk patients with severe aortic stenosis. TAVI has resulted in substantially reduced mortality and morbidity compared to standard treatment methods in the poor surgical risk patient subgroup [2]. It has become a viable option in these patients with increasingly promising short- and mid-term outcomes.

Pre-procedure imaging plays an important role in assessing the anatomy of the aortic annulus, aorta, iliac and femoral arteries in these patients, thereby preventing patient-prosthesis mismatch and determining a suitable vascular approach [3]. Accurate prosthesis-valve matching is necessary not only to reduce the incidence 
and amount of paravalvular regurgitation (if the prosthetic valve is too small), but also aortic rupture (when prosthetic valve is too large). Analyzing the aorta and ilofemoral arteries with attention to size, tortuosity, and calcification determines the optimal prosthetic valve size and access approach. Echocardiography, ECG-gated CT angiography (CTA), and catheter angiography have been used for preoperative planning. Increasingly, CTA is playing an important role towards preoperative assessment of aortic annular dimensions and aortic geometry for optimal prosthetic valve size and functions next to echocardiography in aortic root evaluation. Evaluation of the peripheral vasculature is routinely being performed using helical CTA of the abdomen and pelvis. However, iodinated CT contrast media cannot be administered to patients with severely impaired renal function or severe contrast allergic type reaction [4], and non-contrast CT obtained in such cases is limited in its ability to evaluate non-calcified plaques and luminal dimensions in vessels. Echocardiography is of limited utility in the presence of extensive vascular calcifications or when an optimum acoustic window is not obtainable. In such patients, invasive catheter angiography, transesophageal ultrasound [5], invasive intravascular ultrasound, or low dose catheter CT angiography [6] have been suggested as alternatives for pre-procedure assessment. Aortic annulus sizing for TAVI by transesophageal echocardiography (TEE) also avoids the use of iodinated contrast. However, large annular size measured by CTA or MRI but not TTE is predictive of paravalvular leak [7]. Aortic regurgitation is the most frequent post-procedural complication after TAVI. Any aortic regurgitation is linked with increased late mortality [8].

A subset of patients is increasingly being recognized when the gradient suggests less severe stenosis than the calculated valve area. This can be due to a dilated ventricle with low ejection fraction (EF) or small ventricle with normal EF. In these patients, dobutamine stress echocardiography is used and the aortic valve is considered truly stenotic if the maximum jet velocity rises over $4 \mathrm{~m} / \mathrm{s}$ with dobutamine-induced increase in stroke volume, whereas the AVA remains less than $1.0 \mathrm{~cm}^{2}$ [9].

IRB approval was obtained for this study.

\section{Non-contrast MRI}

Non-contrast MRI is a noninvasive and radiation free diagnostic test that can provide critical pre-TAVI planning information and, thus, replace the use of pre-procedure invasive angiography or CTA. Advantages of MR include the ability to provide both anatomic (annular diameters, perimeter, area) measurements and quantify aortic valve stenosis (flow volumes, peak velocities) with high accuracy [10]. Recent studies have demonstrated that the information gained from both MRI and ECG-gated CTA in the pre-operative assessment of patients undergoing TAVI is reproducible [3, 7, 11-13]. MRI provides numerically similar measurements in terms of annulus size, left ventricular outflow tract (LVOT), and aortic valve area when compared to TTE [11]. MRI can also provide anatomic assessment of the aortic valve for identifying congenital valvular abnormalities, visualization of cardiac structure \& function, and identification of atherosclerotic plaque, aneurysm, or dissection in the ascending aorta.

\section{Emerging indications for MRI}

As the number of TAVI procedures increases, there will also be an increase in the number of patients who cannot be optimally evaluated with CTA, echocardiography, or stress echocardiography. Thus, non-contrast MR may have an important role in preoperative evaluation in the following groups of patients:

1. Patients with history of severe allergic type reaction to intravenous iodinated contrast medium who cannot be administered contrast medium for CTA.

Table 1 Important aortic annular measurements, which identify the optimal Edwards Sapien transcatheter heart valve based on CTA [38] and echocardiography (http://www.edwards.com/eu/products/transcathetervalves/Pages/sapien3.aspx).

\begin{tabular}{llllll}
\hline Valve & $\begin{array}{l}\text { Native Annular size TEE: } \\
\text { Diameter/Area }(\mathrm{mm} / \mathrm{mm} 2)\end{array}$ & $\begin{array}{l}\text { Mean Aortic Annulus } \\
\text { Diameter }(\mathrm{mm})\end{array}$ & $\begin{array}{l}\text { Aortic Annulus } \\
\text { Perimeter }\end{array}$ & $\begin{array}{l}\text { Aortic Annulus } \\
\text { Area (mm2) }\end{array}$ & $\begin{array}{l}\text { Area derived Diameter } \\
\text { Sapien } 23 \mathrm{~mm}\end{array}$ \\
Sapien $26 \mathrm{~mm}$ & $21-25 / 430-546$ & $19-22$ & $60-69$ & $300-380$ & $20.7-23.4$ \\
Sapien $29 \mathrm{~mm}$ & $24-28 / 540-683$ & $23-25$ & $72-78.5$ & $415-490$ & $23.4-26.4$ \\
Sapien3: $20 \mathrm{~mm}$ & $16-19$ & $26-28$ & $81.5-88$ & $530-620$ & $26.2-29.5$ \\
Sapien3: $23 \mathrm{~mm}$ & $18-22$ & & & $273-345$ & $18.6-21$ \\
Sapien3: $26 \mathrm{~mm}$ & $21-25$ & & $338-430$ & $20.7-23.4$ \\
Sapien3: $29 \mathrm{~mm}$ & $24-28$ & & $430-546$ & $23.4-26.4$ \\
\hline
\end{tabular}


Table 2 Important aortic measurement, which identify the optimal CoreValve trans catheter heart valve [38] (http://www.accessdata.fda.gov/cdrh_ docs/pdf13/P130021c.pdf)

\begin{tabular}{llllll}
\hline Valve & $\begin{array}{l}\text { Aortic Annulus } \\
\text { Diameter }(\mathrm{mm})\end{array}$ & $\begin{array}{l}\text { Aortic Annulus } \\
\text { Area }(\mathrm{mm})\end{array}$ & $\begin{array}{l}\text { Aortic Annulus } \\
\text { Perimeter }\end{array}$ & $\begin{array}{l}\text { Ascending Aortic } \\
\text { Diameter }\end{array}$ & $\begin{array}{l}\text { Sinus of valsalva: } \\
\text { Height/Width (mm) }\end{array}$ \\
\hline CoreValve 23 & $18-20$ & $254.5-314.2$ & $56.5-62.8$ & $\leq 34 \mathrm{~mm}$ & $\geq 15 / \geq 25$ \\
CoreValve 26 & $20-23$ & $314.2-415.5$ & $62.8-72.3$ & $\leq 40 \mathrm{~mm}$ & $\geq 15 / \geq 27$ \\
CoreValve 29 & $23-26$ & $415.5-572.6$ & $72.3-84.8$ & $\leq 43 \mathrm{~mm}$ & $\geq 15 / \geq 29$ \\
CoreValve 31 & $26-29$ & $530.9-660.5$ & $81.7-91.1$ & $\leq 43 \mathrm{~mm}$ & $\geq 15 / \geq 29$ \\
\hline
\end{tabular}

2. Impaired renal function (acute kidney injury or chronic kidney injury with serum creatinine $>2 \mathrm{mg} / \mathrm{dL}$, or $\mathrm{GFR}<30 \mathrm{~mL} / \mathrm{min} / \mathrm{m}^{2}$ ).

3. Evaluation of severity of aortic stenosis in patients with poor acoustic window and low cardiac output/low gradient AS (aortic stenosis) with reduced left ventricular ejection fraction (LVEF).

4. Evaluating severity of aortic stenosis in patients with moderate stenosis by echocardiography but symptomatic for severe stenosis and who have contraindications for stress echocardiography (Appendix A) [14].

Post-gadolinium delayed enhanced imaging has been used to assess the severity of coronary artery disease in patients undergoing TAVI, and it has been demonstrated that there is decreased LVEF recovery after TAVI in the presence of significant delayed enhancement prior to TAVI [15]. Use of Gadolinium however is contraindicated in patients with poor renal function. Gadolinium enhanced MRI is not necessary for the preoperative measurements for TAVI.

\section{Advantages of MRI}

Advantages of MRI as compared to CT or catheter angiography include: noninvasive and radiation-free imaging modality, anatomic assessment of the aortic valve for identifying congenital valvular abnormalities, performing planimetry to calculate aortic valve area, visualization of cardiac structures, characterization of the ventricular mass and function, identifying atherosclerotic plaque, aneurysm, or dissection in the ascending aorta. The type and number of available transcatheter aortic valve have evolved and increased over time. Identifying the optimal device and appropriate approach to implant depends on a number of critical measurements.

Although there are publications reviewing the usefulness of MRI, both before and after implantation, for the evaluation of TAVI, these have mostly focused on only a few aspects of the increasingly complex and comprehensive assessment needed for complete assessment prior to TAVI. This article is based on our experience of patients who underwent both contrast enhanced ECG-gated CTA and non-contrast MRI for preoperative assessment for TAVI. It provides a comprehensive review of the key measurements for the pre TAVI assessment as reported on CTA (Tables 1 and 2), most useful acquisition sequences on a $1.5 \mathrm{~T}$ MR scanner (Table 3), and the imaging parameters (Table 4) used for these sequences at our institution.

\section{Limitations of MRI}

There are important limitations of MRI in preoperative assessment for TAVI including multiple breath holds, longer scan

Table 3 Key MR sequence and their utility in pre-operative evaluation for TAVI

\begin{tabular}{|c|c|}
\hline Sequence & Purpose \\
\hline Three-plane localizer & Localize aortic valve plane \\
\hline Axial SSFP non-ECG gated without contrast & $\begin{array}{l}\text { Identify potential ascending aorta and subclavian access sites, } \\
\text { determine size, calcification, and presence of aneurysmal dilatation of aorta }\end{array}$ \\
\hline $\begin{array}{l}\text { Breath held/free breathing 2D ECG-gated SSFP: } \\
\text { Coronal Aorta, LVOT and Aortic Root }\end{array}$ & $\begin{array}{l}\text { Evaluate aortic annulus, aortic valve structure, and sinus height } \\
\text { Planimetry valve orifice area }\end{array}$ \\
\hline SSFP ECG gated images: short axis stack & Calculate ejection fraction, ventricular volumes and mass \\
\hline Breath held/free breathing phase contrast at aortic orifice & $\begin{array}{l}\text { Calculate blood flow velocity, pressure gradient and flow volume across the aortic valve } \\
\text { Calculate aortic regurgitant volume }\end{array}$ \\
\hline 3-D Navigator assisted SSFP & $\begin{array}{l}\text { Coronary ostia height } \\
\text { Aortic diameter }\end{array}$ \\
\hline T2 Black Blood & Useful in presence of susceptibility artifacts from sternal wires or prosthetic valves \\
\hline
\end{tabular}


Table 4 Parameters of the key MRI sequences on a 1.5 T MRI used at our institution

\begin{tabular}{|c|c|c|c|}
\hline Sequence & Flip Angle & $\mathrm{TE} / \mathrm{TR}$ & Slice Thickness/Gap (mm) \\
\hline Axial SSFP non-gated without contrast & 45 & $1.4 / 3.4$ & $6 / 0$ \\
\hline Breath held/free breathing 2D ECG-gated SSFP: Coronal Aorta, LVOT and Aortic Root & 45 & $1.4 / 3.4$ & $5 / 0$ \\
\hline T2 Black Blood & 90 & $41 / 1791$ & $8 / 0$ \\
\hline Breath held/free breathing phase contrast at aortic orifice & 25 & $2.7 / 5.6$ & 8 \\
\hline 3D Navigator assisted SSFP & 75 & $1.8 / 4$ & 2 \\
\hline
\end{tabular}

time, and other contraindications as discussed in appendix B. How well MRI assesses calcified plaques and porcelain aorta is not known. At present, semi-automatic analysis software is available for CT, but none is available for MRI. Not all patients can undergo MRI, and there are certain absolute and relative contraindications for MRI that the TAVI team (clinicians and cardiac imagers) should also be aware of (Appendix B).

\section{Aortic annulus}

The aortic annulus is a virtual ring formed by connecting the basal hinge points of the aortic valve leaflets. The annulus is not a distinct histological entity or anatomical boundary, but represents the smallest diameter in the blood path between the left ventricle and aorta and represents the position for the implanted prosthetic valve [16] (Fig. 1). Bright blood ( $\mathrm{SSFP}=$ steady state free precession) aortic root cine stack of 8-10 slices from diastole to systole is acquired parallel to the valve plane (Movie 1). This plane can be prescribed by planning the acquisition perpendicular to the direction of flow in

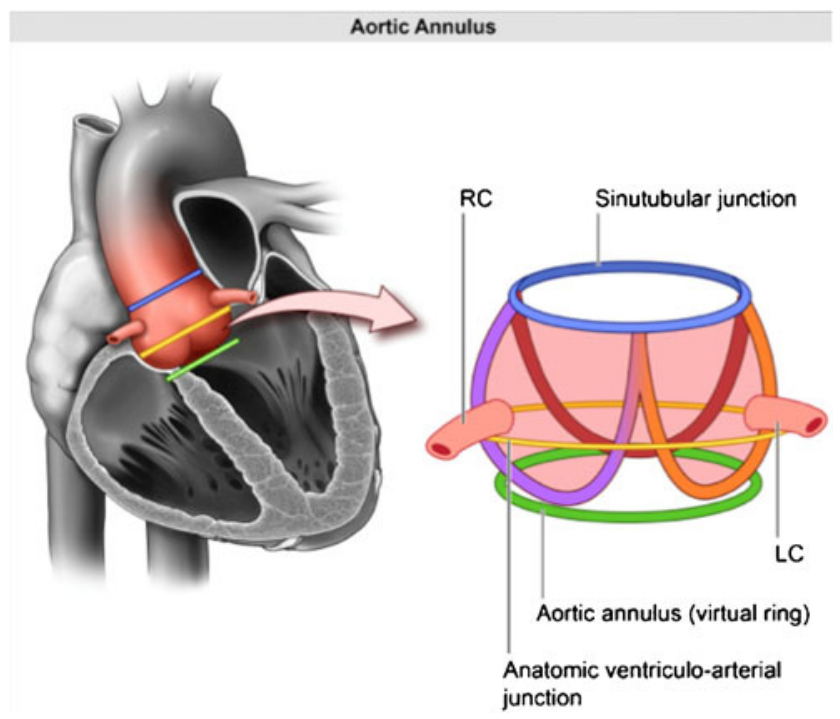

Fig. 1 Pictorial depiction of the aortic root complex demonstrating the location of the annulus, aortoannular, ventriculoarterial and sinotubular junction aortic sinus on these two planes: coronal aorta and left ventricle outflow tract (Fig. 2a, b, respectively). This plane is similar to the suggested plane for performing planimetric assessment of the aortic orifice [11, 13, 17] as well as for performing 2-D phase contrast MRI measurements of aortic flow. Obtaining such a plane ensures that annular area and diameter measurements are obtained perpendicular to the axis of blood flow in LVOT and aorta as has been described for aortic measurements. As there is systolic descent of the annular plane, this acquisition should always begin in the left ventricle outflow tract. The annular plane is identified by the end systolic image below the insertion of leaflets by scrolling through this cine stack (Fig. 2c). This slice is used for assessing the minor \& major diameters, area, and perimeter of the annulus. Annular diameters can also be obtained from a navigator-assisted free breathing diastolic phase 3-D SSFP sequence [3]. Noncontrast 3-D-FLASH MRA has been proposed to obtain diastolic annular dimensions, and using a correction factor based on systolic measurements from CTA in the same patients, a systolic corrected annular dimension can be obtained [18]. Measurement of the annulus is important for correct selection of prosthesis size, type, and to avoid damage of the annulus if the valve is oversized and avoid paravalvular regurgitation if the valve is undersized. Measurements including and excluding calcification are provided. Accurate measurement of heavily calcified annulus remains challenging, and the best method to measure such an annulus has not been determined. In our practice, on both CTA and MRI, heavily calcified annulus area and diameters are measured by including and excluding the calcification. In one study, using an in vivo model of calcium containing rings, MRI measurements have been demonstrated to be the most accurate for assessing the actual dimensions compared to CT or 3-D echocardiography [19]. When there is still uncertainty, measurements obtained by CTA/MRI are compared to annular sizing by calibrated balloon aortic valvuloplasty [20,21]. The degree of annular calcification can also be assessed with MRI; however, how well this assessment is compared to CT is not known. In our experience, a change in signal intensity of the aortic wall should be sought as the presence of calcification leads to a darker interface on SSFP sequences (Fig. 3). 
Fig. 2 Acquisition on MRI for aortic annular plane to measure diameters, area, and perimeter. Aortic root cine stack is prescribed from coronal aorta (a) and left ventricle outflow tract views (b). Systolic image where the luminal diameter is widest, in a location just below the insertion of the valve leaflets (c) is identified as the annular slice. Corresponding annular image from the same patient obtained from ECG-gated CTA demonstrates similar measurement of annular area (d)
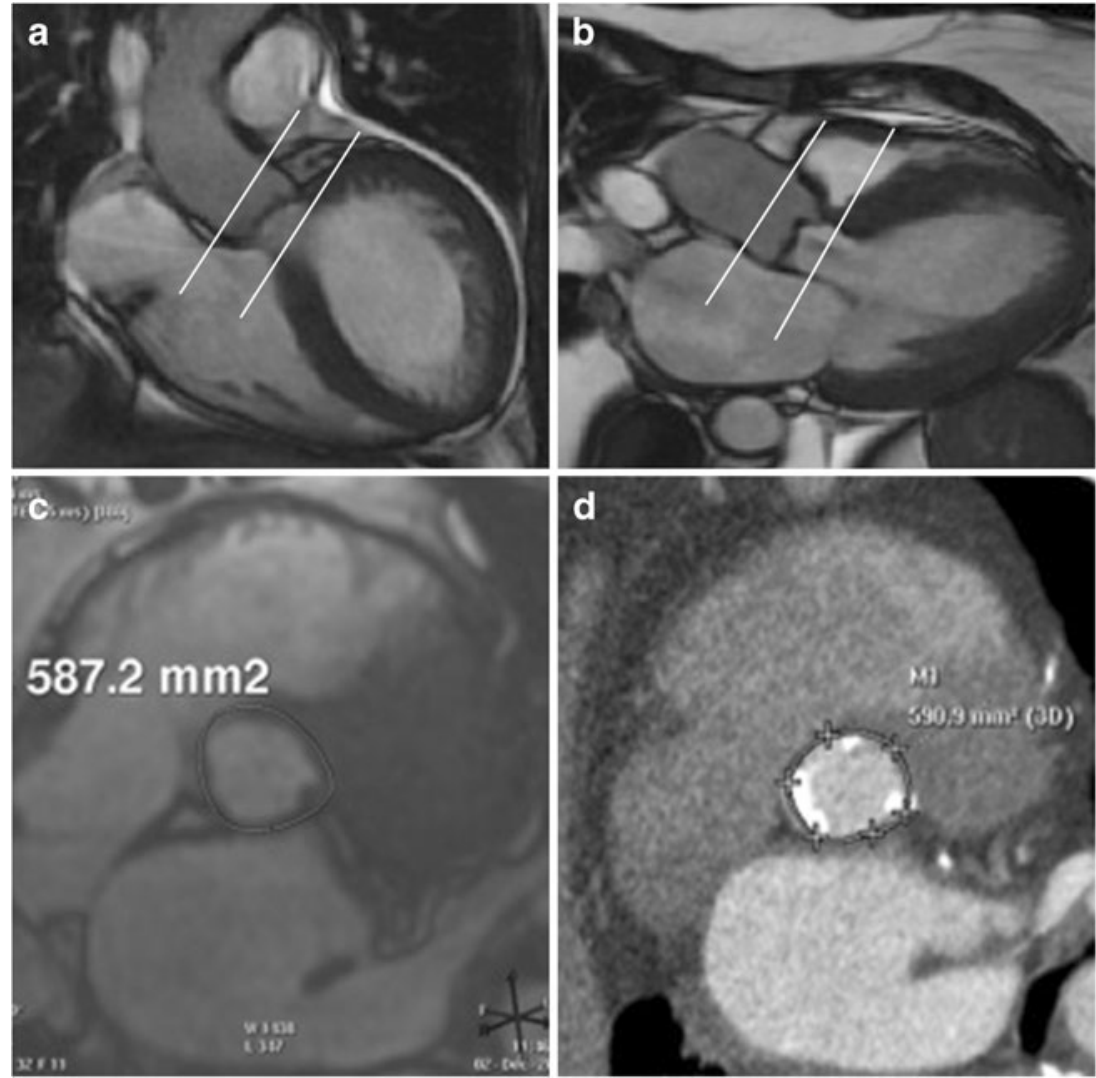

Protruding annular calcifications $>4 \mathrm{~mm}$ and adherent calcification $>6 \mathrm{~mm}$ (particularly left-sided) or calcifications with an Agatston score of $>3000$ [22] are important predictors of paravalvular leaks after TAVI [23]. Comparative CT and MRI studies of heavily calcified annulus and aortic plaques have not been done. In our experience, on MRI, measurement should be obtained from the outer edge to the outer edge. In case of suspected porcelain aorta, a non-contrast CT should be obtained to better characterize the degree and extent of calcification. Evaluation of this aortic root cine stack also helps identify nodular thickening in the valve leaflets, annulus, LVOT, and for the presence of aortomitral continuity calcification/thickening (Fig. 2).

\section{Coronary ostia}

The distance from the annulus to the coronary ostia is of importance to prevent occlusion of the coronary arteries by the displacement of the native aortic valve leaflets by the prosthesis. Risk of coronary ostia occlusion is less with the CoreValve prosthesis than with the Edwards Sapien prosthesis. For the latter, minimum distance values of $10-14 \mathrm{~mm}$ between the coronary ostia and leaflet insertion are usually suggested. A 3-D SSFP free breathing stack obtained in late diastole with a respiratory navigator can be used to assess the height of coronary ostia from the annular plane (Fig. 3a-b, c-d: comparative images from CTA in same patient).

\section{Aortic valve}

Detailed anatomic assessment of the aortic valve can distinguish tricuspid vs. bicuspid, or the quadricuspid valve. TAVI has been successfully performed in selected high-risk patients with severe bicuspid aortic valve stenosis [24]. The severity of aortic stenosis is mostly quantified by transthoracic or transesophageal echocardiogram. In patients with low flow and low gradient but clinically suspected severe stenosis, dobutamine stress TTE is used to augment flow and unmask severe stenosis. Stress TTE may be contraindicated in some patients (Appendix A). Aortic stenosis can be quantified on MRI by different methods. Planimetry of maximal visible valve opening in systole on MRI (Fig. 4) correlate well with planimetry measurements obtained on CT [7, 11, 17]. Severe aortic stenosis is suggested when the aortic valve area is $<1 \mathrm{~cm}^{2}$. The valve leaflets can be evaluated on the 2-D ECG-gated SSFP images (Fig. 5). Stenosis calculation based on the Hakki formula (aortic valve area $=$ cardiac flow $/ \sqrt{ } \max$ imum gradient) correlate well with measurements obtained from echocardiography [25]. In addition, the velocity ratio 
Fig. 3 Assessment of coronary ostial height on MRI. Navigatorassisted 3-D SSFP stack of the aortic root in the late diastolic phase is acquired. Ostial height is measured from the aortic valve annular plane (a: left coronary artery, $\mathbf{c}$ right coronary artery). In the same patient corresponding CT images (b: left coronary artery, $\mathbf{d}$ right coronary artery). This MRI sequence can be also be used to assess sinus of valsalva height and width. Note the dark appearance of the anterior aortic wall and the left ventricle outflow tract due to extensive calcifications, easily seen on the corresponding CTA images
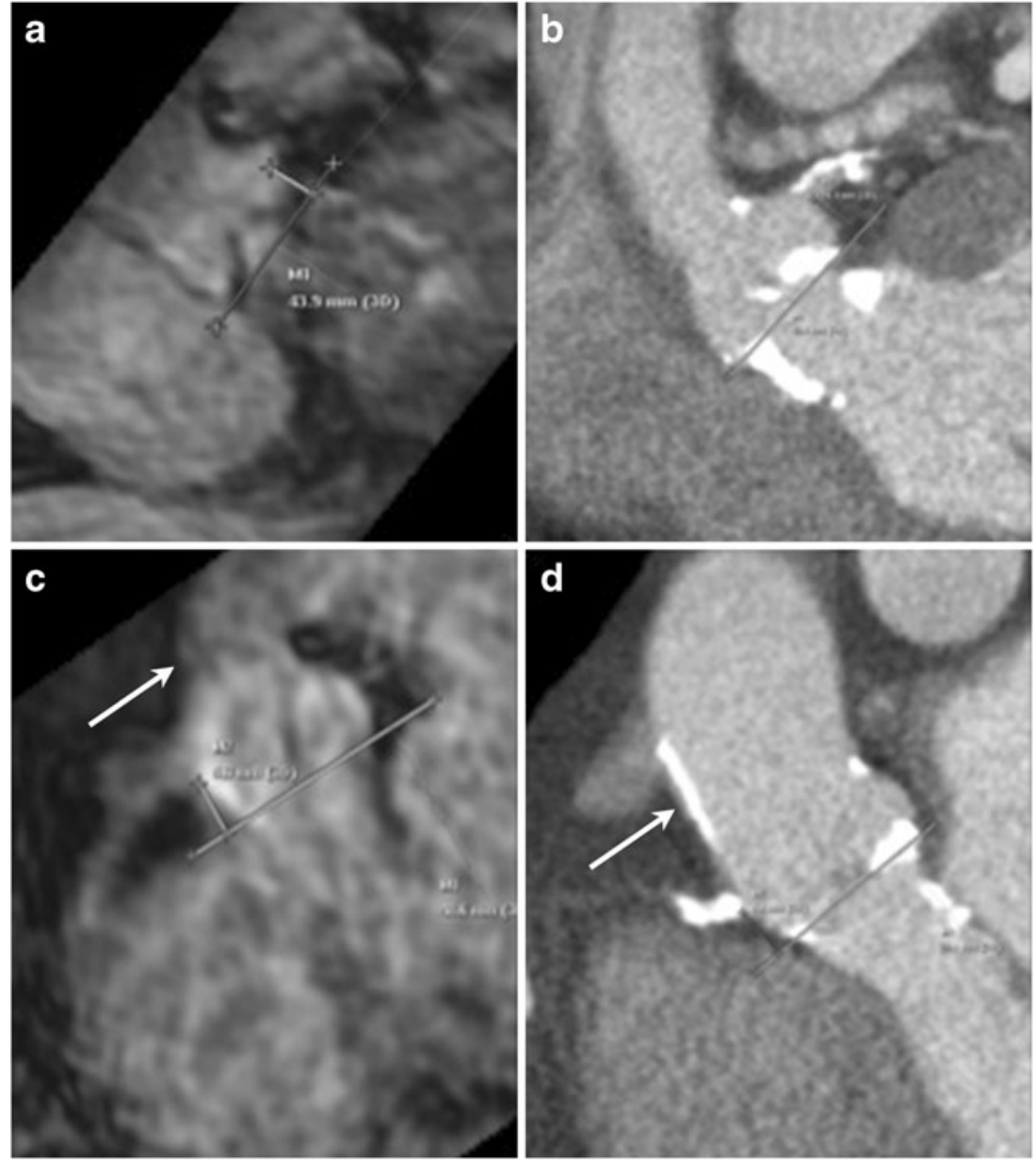

based on peak velocity in the LVOT and aortic sinuses can also be calculated from two separate 2-D phase contrast MR acquisitions. A velocity ratio of $<0.25$ is compatible with severe stenosis.

\section{Aortic sinus}

Measurements of the sinus of valsalva diameters and height are critical for proper positioning of the device to ensure that there is no infringement of the coronary ostia (Tables 1 and 2). Sinus diameter and height measurement can be obtained from breath held or free breathing SSFP sequences (Fig. 5 a-c, e-f: comparable measurements in the same patient from CTA). Sinotubular junction height is critical for proper positioning of the device and verifying no infringement on coronary ostia especially for CoreValve. Susceptibility artefacts can limit evaluation of SSFP sequences in patients who have implantable devices or sternal wires from prior thoracic surgery. In these cases, additional non-fat suppressed T2 black blood images (coronal plane prescribed to aorta for left, and sagittal plane for right coronary artery) can be used to identify sinus diameter, height, etc.

\section{Aortic root}

Aortic root orientation is critical for precise positioning of the device, in particular for the CoreValve. Inappropriate alignment

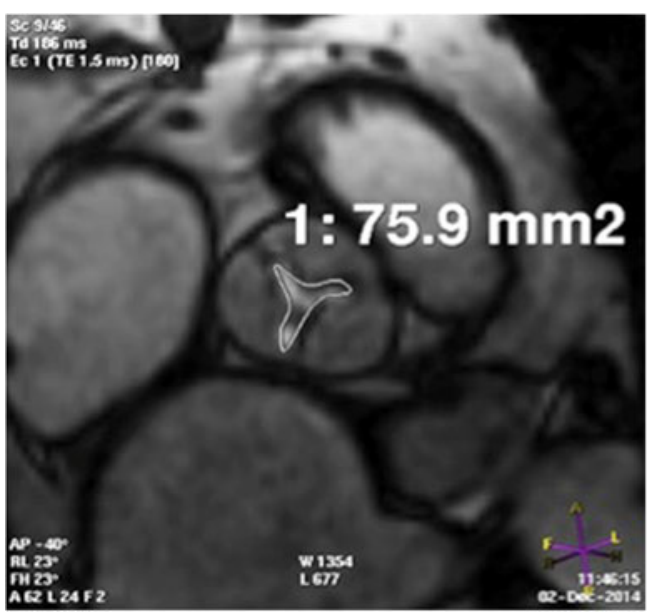

Fig. 4 Assessment of aortic stenosis by planimetry. 2-D cine SSFP acquisition parallel to the valve plane demonstrates the narrowest opening of the aortic orifice. CT of the same patient also demonstrated similar orifice area 
Fig. 5 Diameters of sinuses of valsalva measured on MRI. 2-D SSFP of the aortic root in late diastolic phase is identified from the aortic root stack. Diameters are measured in mid-sinus above the aortic valve (a: noncoronary, b: left and $\mathbf{c}$ right). In the same patient corresponding CT images (d: noncoronary, e: left coronary and $\mathbf{f}$ : right coronary). Navigatorassisted 3-D SSFP or T2 black blood sequences can also be used for these measurements
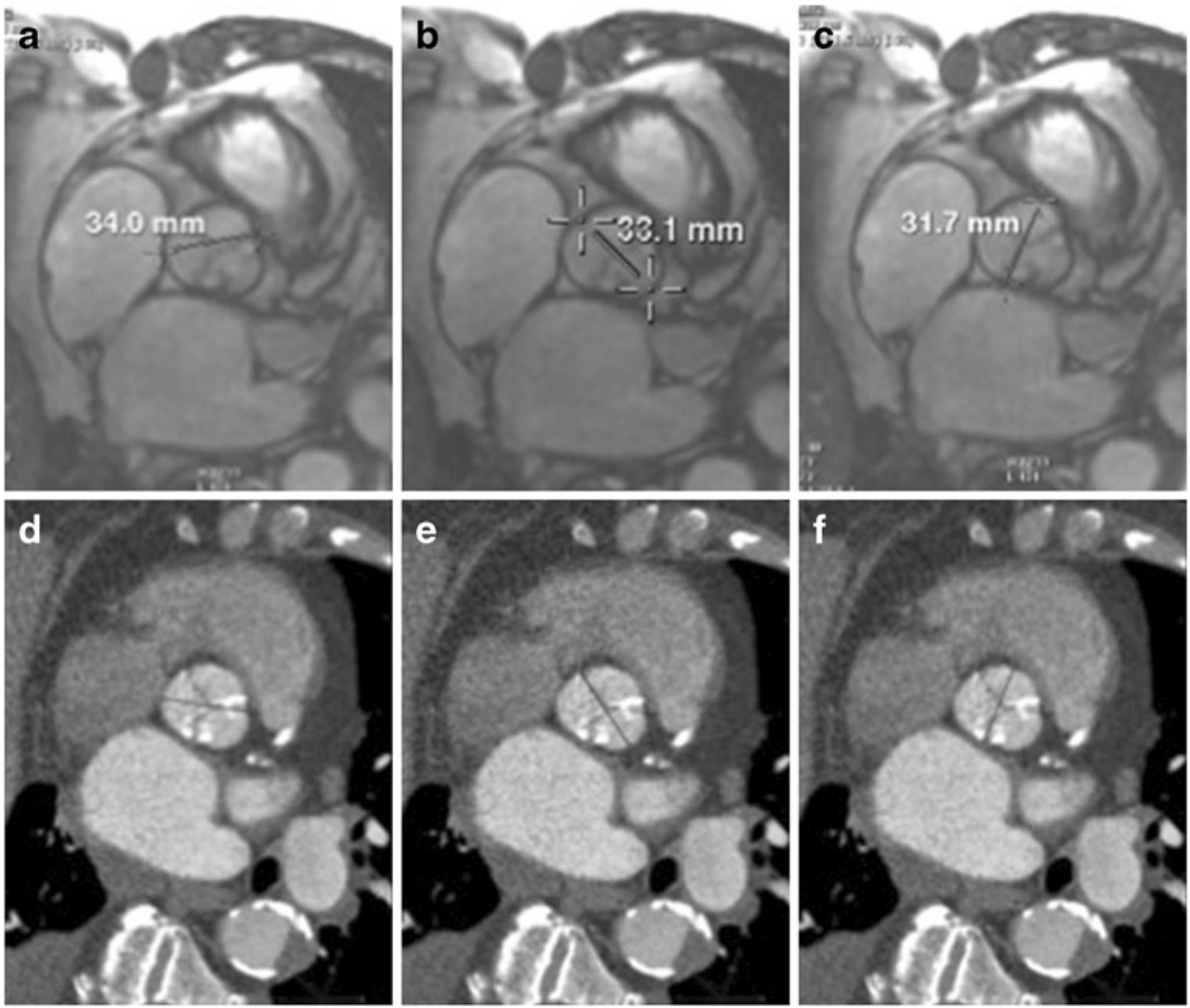

is associated with post-procedural complications such as device malposition or distal embolization. Root angulation can be measured on coronal non-gated SSFP MRI in relation to body axis For example, if the root angle $>30^{\circ}$, a subclavian arterial approach cannot be used for CoreValve implantation [26].

\section{Ascending aorta}

Assessment of the thoracic aorta for plaque burden is important. MRI is not optimal for assessment of aortic calcifications [9]. On MRI, calcifications would lead to signal voids and, hence, appear dark. Silveresa et al., studied carotid and femoral artery plaques using MRI and FDG-PETCT and demonstrated complementary values of these modalities. In this study, lipid-rich plaques were more inflamed than either calcified or collagen-rich plaques. For MRI, they measured the plaque using T2 weighted sequence [27]. Noncalcified atherosclerotic plaque burden of the thoracic aorta may increase the risk for acute renal injury post valve implantation. Aortic wall thickness that exceeded $\geq 2 \mathrm{~mm}$ is defined as a diseased segment [28]. Assessment of plaque thickness between echocardiography and CTA is good [29]. Noninvasive MRI also compares well with TEE for the assessment of atherosclerotic plaque thickness, extent, and composition [30]. Although there are no comparative studies evaluating CT and MRI for thickness of the aortic plaque, plaque thickness should be noted and measured using suggested comparative studies, i.e., distance between the aortic border and the point of greatest luminal protrusion [30].

In addition, evaluating the tubular ascending aorta for any aneurysm or ectasia is important for implanting a CoreValve. Heights of the Edwards Sapien prostheses are between 15$19 \mathrm{~mm}$, and they do not extend beyond the aortic sinus; however, the height of the CoreValve is between $52-55 \mathrm{~mm}$, and these valves extend into the tubular ascending aorta. Therefore, aortic diameter is measured for these at a distance of $40 \mathrm{~mm}$ from the valve plane. Significant aneurysmal dilatation is considered a contraindication for TAVI using this device [21]. All measurements of aortic diameter should be obtained perpendicular to the axis of blood flow [31].

\section{Transaortic access}

The direct transaortic approach is considered as an alternative endovascular access site in patients with unsuitable iliofemoral anatomy. Measurement of the distance from the ascending aorta access site to the skin and valve plane can be performed using axial non-gated SSFP MRI. A favourable puncture site is at the greater curve, typically the right lateral side of aorta with the following criteria: absence of calcification, atheroma, thrombi, and dissection flap or previous surgery [32]. The minimal distance from the aortic annulus to this 
Fig. 6 Identification of access site in tubular ascending aortic for transaortic implantation on MRI. Skin to aortic distance measured on axial non-gated SSFP sequence (a) similar to CTA (b). Access site on aorta to annular plane distance measured from multiplanar sagittal oblique reformats obtained from the same axial non-gated SSFP sequence (c). Same measurement in this patient obtained from CTA $(\mathbf{d})$
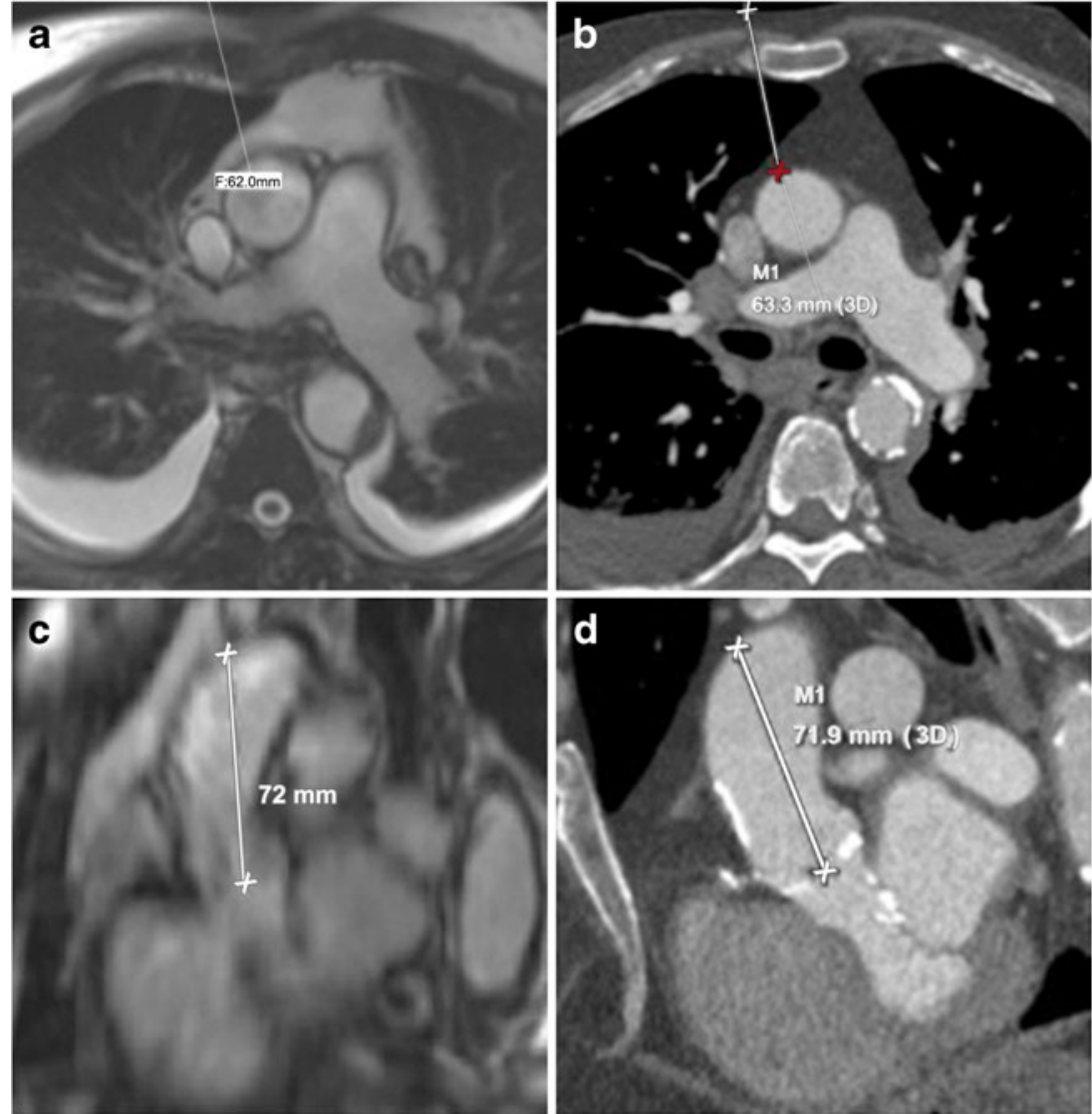

access site needs to be at least $5 \mathrm{~cm}$. This access site needs to be at least $1 \mathrm{~cm}$ distal to coronary venous grafts or foreign bodies (Fig. 6). The relation of the ascending aorta to the sternum helps in identifying the surgical approach. A mini J sternotomy is preferred if the ascending aorta is in midline or toward the left and $>6 \mathrm{~cm}$ deep to the sternum. A mini right thoracotomy is preferred if the ascending aorta is right sided ( $>50 \%$ of the aorta is present on the right of the sternal boarder) at the level of the second intercostal space and is $<6 \mathrm{~cm}$ deep to the sternum [32]. How well are the calcified plaques or porcelain aorta assessed with MRI compared to CT is not known. Presence of calcification may cause loss of signal in the aortic wall (Fig. 3). When porcelain aorta is suspected, a non-contrast CT may be obtained for better evaluation.

\section{Transapical access}

The transapical approach is considered as an alternative access site option for balloon expandable transcatheter valves in patients with unsuitable iliofemoral, subclavian, or aortic anatomy. The left ventricle (LV) apex is evaluated for scar/prior infarct and thrombus. Measurements of the LV apex from the sternum and skin surface can be performed using axial non-gated SSFP MRI similar to CTA (Fig. 7). Advantages of the transapical approach compared to a transfemoral approach include more reliable coaxial alignment of the transcatheter valve with the aortic annulus and greater ability to control delivery of the valve [33].
Fig. 7 Assessment of left ventricle apex on MRI. Location of the apex in the intercostal space and the distance of this access site from the midline can be measured and marked using axial non-gated SSFP sequence (a). Same site location by CTA in this patient (b)
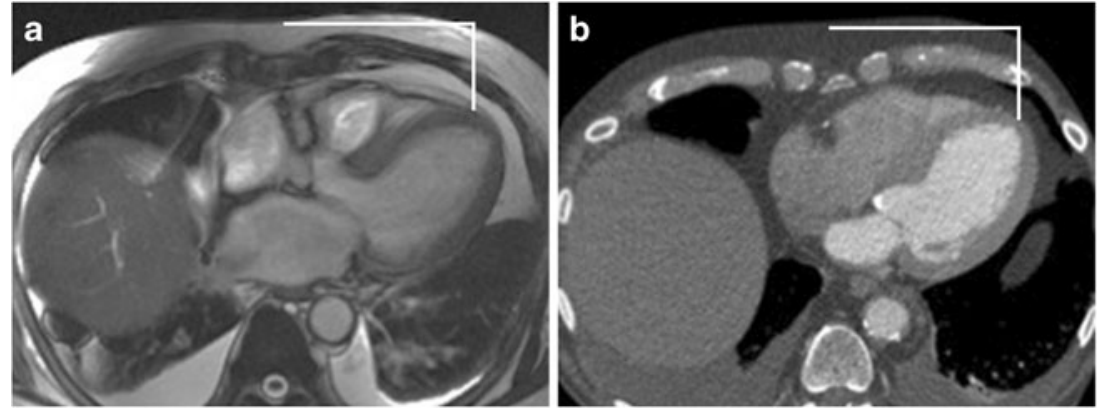


\section{Post TAVI paravalvular regurgitation}

Aortic regurgitation (AR) is the most frequent postprocedural complication after TAVI and is linked to adverse outcomes and mortality. MRI is another quantitative imaging modality that can be used to assess aortic regurgitation after valve implantation. Transthoracic echocardiography displays higher variability and significantly underestimates AV annulus size and underestimates AR when compared to MRI [34, 35]. Compared to echocardiography, quantitative measurement of AR by MRI demonstrates better correlation with quantitative assessment by angiography [36]. MRI may be the modality of choice when there is discordance in grading AR by echocardiography [37].

\section{Conclusion}

Pre-TAVI planning using non-contrast MRI can play a pivotal role in the assessment of the aortic root complex and thoracic access sites in patients who cannot undergo a contrast enhanced CTA either due to severe allergic reaction or severely impaired renal function. Free breathing or navigator-assisted imaging can be performed in patients with dyspnoea. MRI provides hemodynamic information with high accuracy and good correlation with echocardiography. MRI can accurately assess severity of the aortic stenosis when there is a discrepancy between clinical findings and echocardiography (because of low flow, poor acoustic window, or inability to perform a stress study). Anatomic information obtained from MRI is reproducible compared to ECG gated CTA. In the future, further studies may also establish usefulness of non-contrast MRI towards assessment of calcified plaques and peripheral vascular access.

Acknowledgments This study was partly funded by Fischer Fund at Department of Imaging Sciences, University of Rochester.

The authors wish to thank Nadezhda Kiriyak from Department of Imaging Sciences, University of Rochester Medical Center, for contributing original artwork to this article.

\section{Appendix}

A. Contraindications to stress echocardiography are: ventricular arrhythmias, recent myocardial infarction (within 3 days), unstable angina, significant left ventricular outflow obstruction, aortic dissection, and severe hypertension: systolic blood pressure $>180$ diastolic blood pressure $>100 \mathrm{mmHg}$ or symptomatic hypertension [14].

B. Relative contraindications for MRI are: Severe claustrophobia, in which medical sedation is contraindicated or unable to resolve anxiety sufficiently, and life threatening severe arrhythmias. Absolute contraindications are: aneurysm clips, carotid artery vascular clamp, neurostimulator devices, Insulin or infusion pump, implanted drug infusion device, bone growth/fusion stimulator, Cochlear, or ear implant, and ocular foreign bodies (i.e., metal shavings).

Open Access This article is distributed under the terms of the Creative Commons Attribution 4.0 International License (http:// creativecommons.org/licenses/by/4.0/), which permits unrestricted use, distribution, and reproduction in any medium, provided you give appropriate credit to the original author(s) and the source, provide a link to the Creative Commons license, and indicate if changes were made.

\section{References}

1. Bennett CJ, Maleszewski JJ, Araoz PA (2012) CT and MR imaging of the aortic valve: radiologic-pathologic correlation. Radiographics 32:1399-1420

2. Leon MB, Smith CR, Mack M et al (2010) Transcatheter aorticvalve implantation for aortic stenosis in patients who cannot undergo surgery. N Engl J Med 363:1597-1607

3. Quail MA, Nordmeyer J, Schievano S, Reinthaler M, Mullen MJ, Taylor AM (2012) Use of cardiovascular magnetic resonance imaging for TAVR assessment in patients with bioprosthetic aortic valves: comparison with computed tomography. Eur J Radiol 81: 3912-3917

4. (2013) ACR Manual on contrast media

5. Pontone G, Andreini D, Bartorelli AL et al (2012) Aortic annulus area assessment by multidetector computed tomography for predicting paravalvular regurgitation in patients undergoing balloon-expandable transcatheter aortic valve implantation: a comparison with transthoracic and transesophageal echocardiography. Am Heart J 164:576-584

6. Joshi SB, Mendoza DD, Steinberg DH et al (2009) Ultra-low-dose intra-arterial contrast injection for iliofemoral computed tomographic angiography. JACC Cardiovasc Imaging 2:1404-1411

7. Jabbour A, Ismail TF, Moat $\mathrm{N}$ et al (2011) Multimodality imaging in transcatheter aortic valve implantation and post-procedural aortic regurgitation: comparison among cardiovascular magnetic resonance, cardiac computed tomography, and echocardiography. J Am Coll Cardiol 58:2165-2173

8. Kodali SK, Williams MR, Smith CR et al (2012) Two-year outcomes after transcatheter or surgical aortic-valve replacement. N Engl J Med 366:1686-1695

9. Holmes DR Jr, Mack MJ, Kaul S et al (2012) 2012 ACCF/AATS/ SCAI/STS expert consensus document on transcatheter aortic valve replacement: developed in collabration with the american heart association, american society of echocardiography, european association for cardio-thoracic surgery, heart failure society of america, mended hearts, society of cardiovascular anesthesiologists, society of cardiovascular computed tomography, and society for cardiovascular magnetic resonance. J Thorac Cardiovasc Surg 144:e29-e84

10. Rajani R, Hancock J, Chambers JB (2012) The art of assessing aortic stenosis. Heart 98(Suppl 4):iv14-iv22

11. La Manna A, Sanfilippo A, Capodanno D et al (2011) Cardiovascular magnetic resonance for the assessment of patients undergoing transcatheter aortic valve implantation: a pilot study. J Cardiovasc Magn Reson 13:82

12. Koos R, Altiok E, Mahnken AH et al (2012) Evaluation of aortic root for definition of prosthesis size by magnetic resonance imaging and cardiac computed tomography: implications for transcatheter aortic valve implantation. Int J Cardiol 158:353-358 
13. Paelinck BP, Van Herck PL, Rodrigus I et al (2011) Comparison of magnetic resonance imaging of aortic valve stenosis and aortic root to multimodality imaging for selection of transcatheter aortic valve implantation candidates. Am J Cardiol 108:92-98

14. Pellikka PA, Nagueh SF, Elhendy AA, Kuehl CA, Sawada SG (2007) American society of echocardiography recommendations for performance, interpretation, and application of stress echocardiography. J Am Soc Echocardiogr 20:1021-1041

15. Freixa X, Chan J, Bonan R et al (2015) Impact of coronary artery disease on left ventricular ejection fraction recovery following transcatheter aortic valve implantation. Catheter Cardiovasc Interv $85: 450-458$

16. Charitos EI, Sievers HH (2013) Anatomy of the aortic root: implications for valve-sparing surgery. Ann Cardiothorac Surg 2:53-56

17. Lopez-Mattei JC, Shah DJ (2013) When to consider cardiovascular magnetic resonance in patients undergoing transcatheter aortic valve replacement? Curr Opin Cardiol 28:505-511

18. Ruile P, Blanke P, Krauss T et al (2015) Pre-procedural assessment of aortic annulus dimensions for transcatheter aortic valve replacement: comparison of a non-contrast 3D MRA protocol with contrast-enhanced cardiac dual-source CT angiography. Eur Heart J Cardiovasc Imaging. doi:10.1093/ehjci/jev188

19. Tsang W, Bateman MG, Weinert L et al (2012) Accuracy of aortic annular measurements obtained from three-dimensional echocardiography, CT and MRI: human in vitro and in vivo studies. Heart 98: 1146-1152

20. Cerillo AG, Mariani M, Berti S, Glauber M (2012) Sizing the aortic annulus. Ann Cardiothorac Surg 1:245-256

21. Achenbach S, Delgado V, Hausleiter J, Schoenhagen P, Min JK, Leipsic JA (2012) SCCT expert consensus document on computed tomography imaging before transcatheter aortic valve implantation (TAVI)/transcatheter aortic valve replacement (TAVR). J Cardiovasc Comput Tomogr 6:366-380

22. Koos R, Mahnken AH, Dohmen G et al (2011) Association of aortic valve calcification severity with the degree of aortic regurgitation after transcatheter aortic valve implantation. Int J Cardiol 150:142-145

23. Feuchtner G, Plank F, Bartel T et al (2013) Prediction of paravalvular regurgitation after transcatheter aortic valve implantation by computed tomography: value of aortic valve and annular calcification. Ann Thorac Surg 96:1574-1580

24. Wijesinghe N, Ye J, Rodes-Cabau J et al (2010) Transcatheter aortic valve implantation in patients with bicuspid aortic valve stenosis. JACC Cardiovasc Interv 3:1122-1125

25. Puymirat E, Chassaing S, Trinquart L et al (2010) Hakki's formula for measurement of aortic valve area by magnetic resonance imaging. Am J Cardiol 106:249-254

26. Litmanovich DE, Ghersin E, Burke DA, Popma J, Shahrzad M, Bankier AA (2014) Imaging in Transcatheter Aortic Valve Replacement (TAVR): role of the radiologist. Insights Imaging 5: 123-145
27. Silvera SS, Aidi HE, Rudd JH et al (2009) Multimodality imaging of atherosclerotic plaque activity and composition using FDG-PET/ $\mathrm{CT}$ and MRI in carotid and femoral arteries. Atherosclerosis 207: 139-143

28. van Rosendael PJ, Kamperidis V, van der Kley F et al (2015) Atherosclerosis burden of the aortic valve and aorta and risk of acute kidney injury after transcatheter aortic valve implantation. J Cardiovasc Comput Tomogr 9:129-138

29. Tenenbaum A, Garniek A, Shemesh J et al (1998) Dual-helical CT for detecting aortic atheromas as a source of stroke: comparison with transesophageal echocardiography. Radiology 208:153-158

30. Fayad ZA, Nahar T, Fallon JT et al (2000) In vivo magnetic resonance evaluation of atherosclerotic plaques in the human thoracic aorta: a comparison with transesophageal echocardiography. Circulation 101:2503-2509

31. Hiratzka LF, Bakris GL, Beckman JA et al (2010) 2010 ACCF/AHA/AATS/ACR/ASA/SCA/SCAI/SIR/STS/SVM guidelines for the diagnosis and management of patients with thoracic aortic disease: a report of the american college of cardiology foundation/american heart association task force on practice guidelines, american association for thoracic surgery, american college of radiology, american stroke association, society of cardiovascular anesthesiologists, society for cardiovascular angiography and interventions, society of interventional radiology, society of thoracic surgeons, and society for vascular medicine. Circulation 121: e266-e369

32. Bapat V, Attia R (2012) Transaortic transcatheter aortic valve implantation: step-by-step guide. Semin Thorac Cardiovasc Surg 24: 206-211

33. Walther T, Kempfert J (2012) Transapical vs. transfemoral aortic valve implantation: Which approach for which patient, from a surgeon's standpoint. Ann Cardiothorac Surg 1:216-219

34. Orwat S, Diller GP, Kaleschke G et al (2014) Aortic regurgitation severity after transcatheter aortic valve implantation is underestimated by echocardiography compared with MRI. Heart 100:1933-1938

35. Ribeiro HB, Le Ven F, Larose E et al (2014) Cardiac magnetic resonance versus transthoracic echocardiography for the assessment and quantification of aortic regurgitation in patients undergoing transcatheter aortic valve implantation. Heart 100:1924-1932

36. Sherif MA, Abdel-Wahab M, Beurich HW et al (2011) Haemodynamic evaluation of aortic regurgitation after transcatheter aortic valve implantation using cardiovascular magnetic resonance. EuroIntervention 7:57-63

37. Lerakis S, Hayek SS, Douglas PS (2013) Paravalvular aortic leak after transcatheter aortic valve replacement: current knowledge. Circulation 127:397-407

38. Kasel AM, Cassese S, Bleiziffer S et al (2013) Standardized imaging for aortic annular sizing: implications for transcatheter valve selection. JACC Cardiovasc Imaging 6:249-262 